\title{
Das Eine tun und das Andere nicht lassen - Psychoanalyse und Gesundheitswesen
}

\author{
Thomas Merki (Zürich)
}

\begin{abstract}
Zusammenfassung: Psychoanalyse und Gesundheitswesen erscheinen auf den ersten Blick als zwei Felder, die nicht oder zumindest kaum miteinander zu vereinen sind. Auf der einen Seite ein hoch komplexes, interaktives Geschehen zwischen AnalysandIn und PsychoanalytikerIn, welches in einem vorgegebenen Setting, aber in weiten Zeithorizonten der Rahmen darstellt, um individuelles Erleben der Analysanden zu verstehen und mit dem Ziel des Autonomiegewinns zu verändern. Auf der anderen Seite ein hoch reglementiertes gesellschafts- und machtpolitisch bestimmtes Feld, welches klare Rahmenvorgaben gibt und kleinschrittig vorschreibt, wie Heilprozesse unterstützt und realisiert werden sollen. Der Frage, inwiefern diese beiden Felder trotzdem aufeinander bezogen werden können, geht der Autor in seiner Arbeit nach. Anhand und in Abhängigkeit der aktuellen Entwicklungen werden Chancen und Risiken der Psychoanalyse im schweizerischen Gesundheitswesen dargestellt und in einem weiteren Schritt ein möglicher Platz der Psychoanalyse in der Gesundheitsversorgung definiert.
\end{abstract}

Schlüsselwörter: Psychoanalyse, Gesundheitswesen, Salutogenese, Gesundheit, Krankheit, Psychoanalytische Psychotherapie, Gesundheitskosten.

\section{$1 \quad$ Einleitung}

In welchem Verhältnis stehen das hoch komplexe, interaktive Handeln zwischen AnalysandIn und PsychoanalytikerIn zum hochreglementierten gesellschafts- und machtpolitisch bestimmten Feld des Gesundheitswesens? Hier das festgelegte Setting und die weiten Zeithorizonte, welche den Rahmen vorgeben, um das individuelle Erleben des Analysanden zu verstehen. Da die klaren Rahmenvorgaben, wie Heilprozesse unterstützt und realisiert werden sollen. In der Folge werde ich herausarbeiten, inwiefern die beiden Bereiche aufeinander bezogen werden können.

Dabei untersuche ich zuerst die Begriffe Gesundheitswesen und Psychoanalyse bzw. psychoanalytische Therapie. In einem zweiten Schritt beschreibe ich die aktuellen Entwicklungen im Schweizer Gesundheitswesen, um in einem 
weiteren Schritt eine Skizze des Platzes der Psychoanalyse im Gesundheitswesen zu entwerfen.

\section{Die Begrifflichkeiten}

Das Gesundheitswesen eines Landes umfasst alle Personen, Organisationen, Einrichtungen, Regelungen und Prozesse, deren Aufgabe die Förderung und Erhaltung der Gesundheit sowie die Vorbeugung und Behandlung von Krankheiten und Verletzungen ist.

Was aber wird gemeinhin als Gesundheit verstanden? Eine viel zitierte Definition von Gesundheit ist diejenige der Weltgesundheitsorganisation (WHO) vom 22. Juli 1946. Sie lautet: «Gesundheit ist ein Zustand vollkommenen körperlichen, geistigen und sozialen Wohlbefindens und nicht die bloße Abwesenheit von Krankheit oder Gebrechen.»

Eine Krankheit könnte demnach umschrieben werden

, als eine Störung der körperlichen, kognitiven, sozialen und/oder seelischen Funktionen,

, als eine Störung, die die Leistungsfähigkeit oder das Wohlbefinden eines Lebewesens subjektiv oder intersubjektiv deutlich wahrnehmbar negativ beeinflusst

, oder eine solche Beeinflussung erwarten lässt.

Der Soziologe Antonovsky (1997) beschreibt Gesundheit in seinem Modell der Salutogenese nicht als einen Zustand, sondern als einen Prozess. Gesundheit und Krankheit sind für Antonovsky sowohl von Subjektivität geprägte Erlebnisse als auch von objektiven Faktoren bedingte Zustände, deren Ausprägung auf Gesundheits-Krankheits-Kontinuen gedacht werden können. Ein solches Kontinuum könnte zum Beispiel zwischen den Polen des Funktionierens und Nicht-Funktionierens gespannt werden. Je mehr funktionierende Anteile ein Mensch enthält, desto wahrscheinlicher wird er sich auch gesund fühlen. Letzteres ist nach Antonovsky wiederum von anderen Eigenschaften abhängig, z. B. von der Qualität des Kohärenzgefühls oder des Kohärenzsinnes. Der Gesundheitszustand eines jeden Menschen kann theoretisch zu einem beliebigen Zeitpunkt auf diesem Kontinuum lokalisiert werden. Er ist damit jeweils nicht entweder gesund oder krank, sondern mehr oder weniger gesund.

Wenn wir uns jetzt von dieser sozialpsychologischen Betrachtungsweise der Frage zuwenden, welche Anwendungskonzepte die Psychoanalyse selbst verfolgt, dann stossen wir auf sich widersprechende Ansätze. Einerseits kann man die Psychoanalyse als die Mutter der modernen Psychotherapie, aus der sich die 
meisten heutigen Psychotherapieformen heraus entwickelt haben, verstehen. Andererseits war Freuds Haltung ambivalent: Für ihn war die Psychoanalyse Behandlungs- und Forschungsmethode in einem. Die Vorstellung der alleinigen Krankenbehandlung erweckt in der psychoanalytischen Bewegung Unbehagen, da die psychische Störung zur Krankheit wird und die Psychoanalyse in den Sog der Medizin gerät.

Freud (1919a) machte sich schon früh Gedanken über die Möglichkeit, die Psychoanalyse grösseren Teilen der Patienten zugänglich zu machen. Sein Anliegen war es, auch weniger begüterte Menschen in den Genuss einer psychoanalytischen Behandlung kommen zu lassen. In den «Studien über Hysterie» berichtete Freud (1895d) über die Behandlung einer Angsthysterie eines achtzehnjährigen Mädchens in einer Sitzung. Die meisten der Analysen, die Freud in den Anfangsjahren durchführte, könnte man heute als «Kurzzeittherapien» bezeichnen. Interessant dazu ist die Arbeit von Ulrike May (2007). Auch Malan (1965) fand bei der Durchsicht der frühen psychoanalytischen Zeitschriften für die Jahre 1909 bis 1914 sieben Fallberichte über erfolgreiche Kurzpsychotherapien.

Der Pionier auf dem Gebiet der psychoanalytisch orientierten Behandlung ist Wilhelm Stekel, der seine schon 20 Jahre zuvor praktizierten therapeutischen Anschauungen in einem Buch über die «Technik der analytischen Psychotherapie» niederlegte (Stekel 1938).

Auch bei den enger an Freud orientierten Analytikern gab es Versuche, die Behandlungsdauer durch technische Variationen abzukürzen. Ferenczi (1926) versuchte, dieses Ziel durch grössere Aktivität - der Patient sollte über vorgegebene Themen phantasieren, bekam gewisse Auflagen und zeitliche Begrenzungen, der Analytiker übernahm dem Patienten gegenüber bestimmte Rollen - zu erreichen. Diese Ansätze wurden in einer zusammen mit Otto Rank verfassten Schrift (Ferenczi \& Rank 1924) wieder aufgegeben, nachdem sie keine überzeugenden Erfolge brachten.

1946 erschien mit dem Buch von Franz Alexander das erste systematische Werk über psychoanalytisch orientierte Psychotherapie. Die Autoren vertraten dort die Ansicht, das verfügbare psychoanalytischeWissen sei gross genug, um eine Form der Therapie zu entwickeln, die flexibler angepasst sei an die zeitlichen, ökonomischen und individuellen Bedingungen als die Standardmethode. Die Anregungen von Ferenczi, die Übertragung durch Veränderungen der Haltung des Therapeuten und durch Variation von Frequenz und Intervall der Therapiestunden zu beeinflussen, wurden als wertvolle technische Verfahren angesehen. Als den wichtigsten therapeutisch wirksamen Faktor in allen aufdeckenden Psychotherapien bezeich- 
neten die Autoren die «korrigierende emotionale Erfahrung» (corrective emotional experience). Diese besteht darin, dass der Patient im Unterschied zu seinen früheren Erfahrungen in der Elternbeziehung in der Übertragungsbeziehung zu seinem Therapeuten günstigere Erfahrungen macht und zu neuen Lösungen findet. Die corrective emotional experience ist nach der Auffassung Alexanders (1946) wichtiger als Deutungen.

Winnicott (1965/1993) verwies auf Ferenczi (1931), der eine fehlgeschlagene Analyse eines Patienten mit einer Charakterstörung als ein Versagen der psychoanalytischen Technik ansah und nicht als einen Fehler bei der Patientenauswahl. So schreibt Winnicott: «Der hier implizierte Gedanke lautete, die Psychoanalyse könnte lernen, ihre Technik der Charakterstörung oder dem Borderline-Fall anzupassen, ohne sich in Lenkung zu verwandeln und ohne die Bezeichnung Psychoanalyse aufgeben zu müssen» (Winnicott 1965 [1993], 162). Winnicott verweist dann auf die Arbeiten Melanie Kleins, die für eine Anpassung der Technik plädierte, um den psychotischen Störungen in der Kinderanalyse gerecht zu werden. «Dann begann sich das Konzept vom Rahmen der Analyse zu erweitern. Schon Aichhorn (1925) hatte gezeigt, dass man spezielle technische Vorkehrungen treffen muss, wenn der Patient ein antisozialer Fall ist» (Winnicott 1993, 162).

Soweit zu den Begrifflichkeiten.

Ich wende mich nun dem Gesundheitswesen zu und frage nach den aktuellen Entwicklungen.

\section{$3 \quad$ Aktuelle Entwicklungen im Schweizer Gesundheitswesen}

In Zeiten knapper werdender finanziellen Mitteln steigt der Bedarf an empirisch erforschten und abgesicherten, wirksamen psychotherapeutischen Verfahren. Dieser Bedarf wird von den Verhaltens- und Kognitionsforschern mit einer Vielzahl von randomisierten und placebo-kontrollierten Wirksamkeitsstudien befriedigt. Dabei ist klar: ZurWirksamkeit von Psychotherapie zeigen die empirischen Studien positive Befunde (z. B. Smith, Glass \& Miller, 1980; Grawe et al. 1994, Leichsenring \& Leibing, 2003). Es fragt sich nur: «Welche Psychotherapie»? Soll es eine allgemeine Psychotherapie nach Grawe et al. (1994) sein, eine Verhaltenstherapie, von der Margraf (2000a, 2000b) in seinen Studien die besten Resultate verkündet?

In diesem Spannungsfeld muss die Psychoanalyse ihren Platz behaupten oder vielleicht durchaus neu definieren und erkämpfen.

Die folgenden Entwicklungen im Gesundheitswesen lassen sich stichwortartig festhalten und verweisen darauf, dass der Verteilungskampf in der Gesundheitsversorgung insgesamt härter wird: 
1. Es findet ein Leistungsabbau statt. Die Krankenkassen übernehmen weniger Kosten für Psychotherapien.

Die auf den 1. Januar 2007 in Kraft gesetzte Änderung der Krankenpflege-Leistungsverordnung (KLV) bringt neu die Meldepflicht von psychotherapeutischen Behandlungen deren Dauer mehr als 10 Stunden beansprucht. Mit der Meldung muss eine Kostengutsprache für die weitere Dauer der Behandlung beantragt werden. Nach 40 Stunden, früher nach 60, muss dem Vertrauensarzt Bericht erstattet und ein begründeter Vorschlag für die allfällige Fortsetzung der Behandlung gemacht werden. Die Änderungen der KLV werden von einer Arbeitsgruppe begleitet und extern evaluiert.

Die bisherigen Erfahrungen zeigen, dass die geänderte Verordnung von den Leistungserbringern ohne größere Probleme adaptiert wird. Auffallend ist, dass bis heute nur wenige 40-Stunden-Berichte eingegangen sind.

2. Präzise Aussagen zur Dauer der Behandlung werden nach wenigen Stunden verlangt. Nebst dem berechtigten Einwand, dass präzise Aussagen zu einem solch frühen Zeitpunkt seriöserweise nicht möglich sind, bedeutet dies vor allem eine Erhöhung des Legitimationsdruckes bei den Leistungserbringern.

3. Die Effizienz von Behandlungen wird im Vergleich zu früher höher gewichtet. Das Ziel sind möglichst kurze Behandlungen.

4. Berufsausübungsbewilligungen werden restriktiver gehandhabt

5. Die delegierte Psychotherapie ist stärker reglementiert. In der ab dem 1. Januar dieses Jahres gültigen Tarmed-Version ist die delegierte Psychotherapie verbindlich geregelt. Demnach wird auf Seiten des Arztes der Nachweis der fachlichen Dignität verlangt. Auf Seiten der delegiert arbeitenden Psychotherapeuten wird ein abgeschlossenes Hochschulstudium in Psychologie und eine anerkannte psychotherapeutische Weiterbildung verlangt.

6. Ein Gesetz über die psychologischen Berufe ist in Vorbereitung: Mit dem Prozess der Gesetzesentwicklung im psychologischen Berufsbereich wurde ein neuer Grad von Professionalität erreicht, der von allen Beteiligten grundsätzlich begrüßt und akzeptiert wird. Ging es ursprünglich in der Gesetzeskommission Fleiner um ein Psychotherapie-Gesetz, was an der Uneinigkeit der beteiligten psychotherapeutischen und psychologischen Berufsverbände und am entschiedenen Widerstand der ärztlichen Standesorganisationen scheiterte, geht es in der aktuellen Gesetzesvorlage 
auch um die Regelung einer Vielzahl von psychologischen Berufen, von denen der eine die Psychotherapie ist. Man kann sich mit Fug und Recht fragen, ob die Psychotherapie nun zu einer Unterdisziplin der Psychologie geworden ist. Hier sind sicher ein großes Konfliktpotenzial zwischen den verschiedenen Interessenvertretern und ein Mischmasch von mehr oder minder schützenswerten Patienteninteressen zu finden. Erwünscht ist aber der im Gesetzesentwurf vorgesehene einheitliche Standard bezüglich Grundausbildung und psychotherapeutischer Weiterbildung der Psychotherapeutinnen.

Oftmals wird im Zusammenhang mit diesen Entwicklungen betont, dass sich die Kosten im Gesundheitswesen in der Schweiz massiv erhöht hätten, so dass es notwendig sei, das Feld restriktiver zu definieren: Das heißt, wie schon erwähnt, dass sich die psychotherapeutische Arbeit gegenüber den Leistungsträgern stärker legitimieren muss. Dabei wird von dieser Seite vorausgesetzt, dass die Behandlungsdauer möglichst kurz angesetzt wird.

Die Analysen von Interpharma (Verband der forschenden pharmazeutischen Firmen der Schweiz) zeigen aber, dass das Argument der explodierenden Kosten im Gesundheitswesen so einfach nicht stimmt. Der Verband schreibt in seinem statistischen Jahrbuch «Das Gesundheitswesen in der Schweiz Leistungen, Kosten, Preise» (Ausgabe 2007) im Kapitel Struktur und Kosten des Gesundheitswesens:

Im Jahr 2005 betrugen die gesamten Ausgaben für das Gesundheitswesen rund 52.7 Milliarden Franken. Zwischen 1996 und 2005 sind die Gesundheitskosten um 39 Prozent angestiegen, während sich die Prämien in der Grundversicherung im gleichen Zeitraum viel stärker erhöht haben (+67.5 Prozent). Nachdem die Krankenkassenprämien vor 2003 stark angestiegen sind, haben sie sich in den letzten Jahren etwas stabilisiert. Die Ausgaben für das Gesundheitswesen sind zwischen 1996 und 2005 prozentual stärker gewachsen als das Bruttoinlandprodukt. Diese Entwicklung war auch zwischen 1985 und 1995 zu beobachten. Im Jahr 2005 stiegen die Gesundheitskosten weniger stark als im Vorjahr, nämlich um zwei Prozent. Der im Gegensatz zur Beobachtungsperiode 1990-1992 etwas abgeflachte Anstieg der Gesundheitskosten verläuft nahezu linear. Von einer «Explosion» der Gesundheitsausgaben kann aufgrund dieser Entwicklung nicht gesprochen werden (Interpharma 2007: 58).

Zudem sind die Kosten für Psychotherapie insgesamt sehr gering. Im Jahr 2000 (das sind diesbezüglich die aktuellsten vorhandenen Zahlen!) betrugen die Gesamtkosten der ambulanten Psychotherapie in der Schweiz 579 Mio. SFr. 
(Sturny u. Hell 2007), also rund 1\% der Gesamtkosten der Aufwendungen für das Gesundheitswesen. Davon bezahlte die Grundversicherung der Krankenkassen 396 Mio. SFr. oder 68\% der Gesamtkosten (Beeler et al. 2003).

Ein weiterer Aspekt ist folgender:

Anfangs der neunziger Jahre begannen sich die Krankenkassen offensichtlich auch im Grundversicherungsbereich weg vom sozialen Krankenkassenmodell hin zum Versicherungsmodell zu entwickeln. Obwohl die Krankenkassen im Rahmen der sozialen Grundversicherung jede Person vorbehaltlos aufnehmen müssen, begannen einzelne Kassen mit Tricks und Kniffs schamlos, Jagd auf sogenannte «Gute Risiken» zu machen. Eine möglichst schnelle und kostengünstige Fallabwicklung steht einseitig im Zentrum. Die von Brunner, damaliger VizeDirektor des Bundesamt für Gesundheitswesen BAG, im Jahr 2005 ausgestoßene Drohung, die psychotherapeutischen Behandlungen aus dem Leistungskatalog der Grundversicherung zu kippen, steht auch in diesem Zusammenhang und hatte meiner Meinung nach den Zweck, den Legitimationsdruck unter den Leistungserbringern zu erhöhen.

Diese Entwicklungen bilden den gesundheitspolitischen Rahmen, der die Psychotherapie und insbesondere die Psychoanalyse maßgeblich beeinflusst. Zusammengefasst sind es insbesondere zwei Punkte:

- Einseitige, kurzfristige Kosten-Nutzen-Überlegungen, anstatt langfristige Konzepte für die Behandlung von Patienten mit psychischen Störungen

- Die Patientin steht je länger desto weniger im Zentrum des Interesses.

\section{Der Platz der Psychoanalyse in der Gesundheitsversorgung - eine Skizze}

Die Beantwortung der Frage nach dem Platz der Psychoanalyse im Gesundheiswesen dürfte sich auch in der Schweiz aus der Praxis ergeben. Aus der Praxis, weil die Psychoanalyse allen Anfeindungen zum Trotz, nach wie vor zu den wissenschaftlich anerkannten Verfahren gehört!

Cremerius (1990: 8-9) beschreibt die Situation in Deutschland und kommt unter anderem zu folgendem Schluss: "Die psychoanalytische Einzeltherapie ist das am häufigsten beantragte Verfahren. Es macht 40\% aller Anträge aus. Es wird jedoch von etwa der Hälfte aller Antragssteller nur zweistündig angewandt. Die zweistündige Einzeltherapie stellt damit so etwas wie ein neues Standardverfahren dar.» Weiter führt Cremerius in der selben Arbeit aus «... dass sich die Analytiker überall in der Welt, wo der Patient, bei dem das analytische Langzeitverfahren indi- 
ziert oder der eine hochfrequente Analyse bezahlen kann, selten wird oder gar aus ihren Praxen verschwindet, an diese Verhältnisse anpassen» (ebd.: 8-9).

Von der Arbeit dieser Kollegen nehme die institutionalisierte Psychoanalyse aber keine Kenntnis, weil sie für sie nicht exisistiere. Erstaunlich, da diese Gruppe die Majorität bildet, so Cremerius.

\section{Exkurs: Was unterscheidet eigentlich den heutigen potenziellen Patienten von demjenigen von 1918? Die ökonomische Situation? Anderes?}

Mit Bezug auf Helmut Thomä und Horst Kächele (1996) kann konstatiert werden, dass sich dieWelt seit den 30er Jahren des letzten Jahrhunderts tiefgreifend verändert hat. Die Verunsicherung des Atomzeitalters und der Globalisierung von Wirtschaft, Information usw. wirken über die Auflösung von familiären und sozialen Strukturen auf den Einzelnen ein. Historische und psychosoziale Prozesse, die sich so auf das Familienleben auswirken, dass sich beim Einzelnen seelische oder psychosomatische Erkrankungen herausbilden, ziehen sich oft über Generationen hin. Unbewusste Einstellungen wie sie mit ihren typischen Inhalten in Familien tradiert werden, folgen andererseits den Regeln des Familienromans. Zwischen traditionellen familiären Traditionen und historischen gesellschaftlichen Prozessen ergeben sich so beträchtliche Asynchronizitäten. Nach Thomä und Kächele (1996, 53) ergeben sich daraus drei Hypothesen:

, «Die sexuelle Revolution hat die Verdrängung der Sexualität insgesamt verringert und die Pille hat die Emanzipation der Frau entscheidend gefördert und ihr mehr Selbstbestimmung in der Geschlechterrolle ermöglicht.

, Hysterische Erkrankungen sind - der Voraussage der psychoanalytischen Theorie entsprechend - seltener geworden.

, Ödipale Konflikte scheinen heutzutage eher zu persistieren als dass sie sich komplexhaft zum Über-Ich strukturieren.»

Was sind die Auswirkungen dieser Aussagen?

Die typische, familiäre Entstehungsgeschichte der seelischen Erkrankungen mit besonderer Berücksichtigung der Kindheit war der Gegenstand der psychoanalytischen Untersuchungsmethode. Die psychosozialen Einflüsse auf die Adoleszenz, die dem Jugendlichen nach Peter Blos (1985) «eine zweite Chance» geben, wurden unterschätzt. Gleichzeitig wurden symptomaufrechterhaltende Faktoren eher wenig berücksichtigt. Weil die frühe Es-Analyse und die spätere ich-psychologisch begründete Widerstandsanalyse von früh erworbenen, stabilen bis rigiden Strukturen ausgehen konnte, wirkte sich dies nur wenig aus. 
Einen Kontrapunkt zur Auflösung psychosozialer und historischer Strukturen stellt nach Thomä und Kächele (1996) das in den Mittelpunkt gerückte Thema der Sicherheit dar. Das Thema der Sicherheit nimmt erst in der heutigen Zeit einen grossen Platz in der Diskussion über die psychoanalytische Behandlungstechnik ein. Die Anfänge sind aber über die 30er Jahre zu Freud und Adler zurückzuverfolgen. In diesem Zusammenhang kann nach Thomä und Kächele (1996) auch die Wirkung von Heinz Kohut's Innovation gesehen werden. Patient und Analytiker sind gleichermaßen mit den konfliktpsychologischen Konzepten unzufrieden und suchen nach Ganzheit und Bestätigung, also nach narzisstischer Befriedigung.

Dieses mit sich selbst Eins-Sein wird offensichtlich mehr und mehr im Einklang mit dem Wir gesucht. Das Menschenbild in der Therapie verschiebt sich vom Bild des abgegrenzten individuellen psychischen Apparates hin zu einem psychosozialen Geflecht der universalen Gegenseitigkeit. Von der Austauschbeziehung zwischen Mutter und Kind, die einst von der ungarischen PsychoanalyseSchule in den Mittelpunkt gerückt wurde, führt die Spur weiter zu neuen psychosozialen Menschenbildern, durch die sich Psychoanalyse, Gestalttherapie, Familientherapie und Körpertherapie anscheinend annähern. Die Therapeuten öffnen sich mehr partnerschaftlich in der Therapie bzw. entdecken ihre persönliche Teilnahme an den Prozessen, die sie, dem Abstinenzdogma folgend, vielfach in der Bedeutung unterschätzt hatten (Richter, 2005). Aber diese Öffnung erschließt ihnen auch mehr die persönliche Einbettung in die geistigen, ökonomischen und politischen Prozesse ringsum und macht ihnen zudem die eigene Mitverantwortung fühlbar.

\section{Wie verhält sich nun die Psychoanalyse zu den skizzierten Entwicklungen im Gesundheitswesen?}

Hier zeigen sich zum einen ein paar Divergenzen, die den Platz der Psychoanalyse im Gesundheitswesen durchaus schwierig machen:

1. Die Psychoanalyse ist insofern «antiquiert», als dass sie keine Rezepte verspricht. Im Gegenteil, sie hat den Menschen in all seinen Facetten im Focus, versucht zu verstehen und zu deuten. In diesem Sinne ist sie eine radikale Absage an einen «modernen» Machbarkeitsglauben.

2. Die Psychoanalyse unterscheidet sich nach Fitzgerald Crain (2005) weiter durch die im Idealfall aufgehobene Trennung zwischen dem «gesunden» Subjekt der Therapeutin oder des Therapeuten und dem «kranken» Objekt der Patientin oder des Patienten. Um einen kranken Menschen zu verstehen, muss der Pschoanalytiker/die Psychoanalytikerin seine Depression, 
seine Zwangsgedanken, sein Gewaltpotential in sich selbst wieder finden. Es gibt keine Reflexion ohne Selbstreflexion, kein Verständnis von Übertragungsprozessen ohne Verständnis von Gegenübertragungsgefühlen. So gesehen ist die Psychoanalyse eine unbequeme und gesellschaftspolitische Wissenschaft; sie konfrontiert einen mit der eigenen Person und den eigenen Schattenseiten, im Gegensatz zu anderen Verfahren und der akademischen Psychologie, die dazu neigen den «allgemeinen Menschen», losgelöst von seinen sozialen Bezügen, zu untersuchen (Crain, 2005). Die Existenz eines «allgemeinen» Menschen wird von der Psychoanalyse bestritten. Sie begreift den Menschen immer als Teil seiner Umwelt und ist deshalb immer «politisch». Neuro-biologische Theorien, welche einfache Erklärungsmuster liefern und einfache Abhilfe versprechen, z. B. psychopharmakologische, liegen heute im Trend. Gegenüber diesen Positionen verkörpert die Psychoanalyse einen gegensätzlichen und unbequemen Standpunkt.

3. Seelische Erkrankung erfasst immer den Menschen als Ganzes. Deshalb ist das Ziel einer psychoanalytischen Behandlung nicht nur die Symptomheilung, sondern auch eine strukturelle Persönlichkeitsveränderung im Sinne größerer innerer Erlebnis- und Denkfähigkeit. Die pathologische psychische Organisation unterliegt häufig äußerst komplexen Bedingungen, welche lange therapeutische Prozesse erfordern können.

Soll der selbstunsicheren Patientin ein Kurs zur Erlangung der Selbstsicherheit verschrieben werden oder ihre Selbstunsicherheit im lebensgeschichtlichen Kontext verstanden werden? Natürlich soll die psychoanalytische Psychotherapie der Patientin zu vermehrter Autonomie und Selbstsicherheit verhelfen, aber Menschen können ihre Identität nicht einfach ablegen und gegen eine neue eintauschen.

Auch in der Psychoanalytischen Kurzzeittherapie, die sich bei klar umschriebenen Problemen und akuten Belastungsreaktionen eignet, wird ein vertieftes Verständnis der unbewussten Zusammenhänge der aktuellen Erkrankung gefördert. Die Kurzzeittherapieverfahren stossen jedoch an ihre Grenzen bei schweren und chronischen Krankheitsbildern und zwar, wie Blatt et al. (2000) und Elkin (1994) untersuchten, sowohl bei kognitiven verhaltenstherapeutischen, wie auch bei interpersonalen Therapieverfahren. Wie verschiedene Untersuchungen belegen, können dagegen Patienten den Effekt Psychoanalytischer Langzeittherapien durch die erzielte Persönlichkeitsveränderung über das Therapieende hinaus noch steigern (DPV 2001). 
Ist es also möglich, trotz der immer stärker werdenden Reglementarien die zentralen Instrumente, Konzepte und Ansatzpunkte in der psychoanalytischen Behandlung aufrechtzuerhalten (- ohne sich selber zu verkaufen)? Ich meine ja.

So ist die zentrale Brücke zwischen den Konzepten der Psychoanalyse und jenen des Gesundheitswesens das Ziel, psychische Störungen bei Menschen zu behandeln. Zudem kann man sowohl in der Gesundheitsversorgung wie auch in der Psychoanalyse die Suche nach Behandlungsmethoden beobachten, die kürzer, kostengünstiger und trotzdem effektiv sein können. Gerade der Blick in die Vergangenheit zeigt,

, dass die Psychoanalyse immer hinterfragt worden ist hinsichtlich ihres effektiven methodischen Vorgehens und hinsichtlich ihrer Stärken und Schwächen,

, dass es das stabile Bild, wie man PatientenInnen behandeln soll, gar nicht gibt,

, dass man sich immer auseinandergesetzt hat mit der optimalen Passung zwischen Theorie, Patient und Methode,

, dass es immer wieder notwendig gewesen ist, sich gegenüber neuen Erkenntnissen und Verfahren offen zu halten und die eigene Arbeit auch immer wieder neu zu legitimieren, und zwar nicht nur aus einer ökonomisch bedingten Notwendigkeit heraus, sondern weil dies ein zentrales Merkmal wissenschaftlichen Arbeitens ist und nur so wissenschaftlicher Fortschritt möglich ist.

Die Realität der Praxis zeigt zudem, dass dies die AnalytikerInnen weitestgehend bereits so praktizieren und ihre Behandlungsmethoden an die neuen Krankenbilder und Kontextbedingungen anpassen (vgl. dazu die Ergebnisse von Cremerius). In diesem Sinne ist zu fragen, ob der im Gesundheitswesen stattfindende Leistungsabbau nicht nur als negativ zu begreifen ist, sondern auch als Chance verstanden werden kann, darüber nachzudenken, wie lange Behandlungen sein sollen, wie hoch die Stunden-Frequenz sein soll oder wann bestimmte Ziele der Behandlung als erreicht gelten können. Im Zentrum stünden dann die Fragen des Verhältnisses zwischen Effizienz (möglichst kurz, kostengünstig) und Effektivität (Grad der Erreichung der Ziele). Dies ist aber aus meiner Perspektive nicht nur die zentrale Frage im Rahmen der Gesundheitsvorsorge, sondern auch eine zentrale Frage im psychoanalytischen Diskurs.

Bedeutsam ist zudem, dass die Kenntnis vom Unbewussten, das Wissen um und über das Übertragungs- und Gegenübertragungsgeschehen die Psychoanalyse 
von anderen Psychotherapieverfahren abhebt und ihr deshalb einen besonderen Platz in der Gesundheitsversorgung gibt.

Unser Potential ist die Kenntnis von den unbewussten seelischen Zusammenhängen, die Fähigkeit, die sich entfaltende Dynamik zwischen TherapeutIn und PatientIn zu verstehen und zu interpretieren und therapeutische Beziehungen unter schwierigen psychodynamischen Bedingungen aufzubauen, zu gestalten und über längere Zeiträume aufrecht zu erhalten. So kann beispielsweise aggressives Ausagieren von destruktiven Impulsen manchmal verhindert oder eingedämmt werden. Dies wirkt einer Somatisierung und Invalidisierung entgegen und hilft auch, Kosten einzusparen.

Wir PsychoanalytikerInnen sollten unser Wissen und Potential zur Behandlung von psychischen Störungen vermehrt einer weiteren Öffentlichkeit bekanntmachen. Gleichzeitig müssen wir Abschied nehmen vom Verständnis der alleinigen Kompetenz bei der Behandlung von psychischen Störungen und interdisziplinär mit anderen Richtungen der psychotherapeutischen Behandlungskette zusammen arbeiten, wo dies angezeigt und möglich ist.

In der heutigen Zeit, die geprägt ist von einem gesellschaftlichen Umverteilungskampf von unten nach oben, gerät das Gesundheitswesen unter starken Kostendruck. Es zählt nicht mehr der Grundsatz, dass die Gesunden für die Kranken bezahlen, sondern der Gesunde soll möglichst wenig bezahlen. Die daraus entstehende Entsolidarisierung bereitet den Boden für die genannten Abbauszenarien vor. Dass dabei Therapieverfahren, die auf einem rein abstrakt-manualisierten Verfahren mit hoher Ergebnisqualität in kürzester Zeit basieren, Hochkonjunktur feiern, wird überaus klar. Wir haben dieser Tendenz jedoch etwas entgegenzusetzen: Das Wissen um die Vielfalt der menschlichen Seele und deren Störungen, die Fähigkeit der Gestaltung von therapeutischen Beziehungen und die Fähigkeit, komplexe Situationen zu verstehen und auszuhalten. Deshalb wird die Psychoanalyse als Heilbehandlung in angewandter Form ihren Platz behalten, wenn wir sie denn nicht selbst abschaffen.

\section{$7 \quad$ Literatur}

Alexander, F. (1946): Psychoanalytic therapy. New York: Ronald Press.

Antonovsky, A. (1997): Salutogenese. Zur Entmystifizierung der Gesundheit. Tübingen: dgvt-Verlag.

Beeler, I; Lorenz, S.; Szucs, T D. (2003): Provision and remuneration of psychotherapeutic services in Switzerland. Sozial- und Präventivmedizin, 48, 88-96. 
Blatt, S., Zuroff, D., Bondie, C. und Sanislow, C. (2000): Short- and long-term effects of medication and psychotherapy in the brieftreatment of Depression: further analysis of data from the NIMH TDCRP. Psychotherapy Research $10,215-234$

Blos, P. (1985): Son and father. Before and beyond the Oedipus complex. New York: Free Press.

Crain, F. (2005): Fürsorglichkeit und Konfrontation. Psychoanalytisches Lehrbuch zur Arbeit mit sozial auffälligen Kindern und Jugendlichen. Giessen: Psychosozial-Verlag.

Cremerius, J. (1990): Die hochfrequente Langzeitanalyse und die psychoanalytische Praxis. Utopie und Realität. In: Psyche - Zeitschrift für Psychoanalyse und ihre Anwendungen, 44(1), 1-29.

DPV (2001): Indikation und Wirksamkeit. Psychoanalyse und psychoanalytische Verfahren in der medizinischen Versorgung. Informationsschrift. Berlin: Deutsche Psychoanalytische Vereinigung.

Elkin, I. (1994): The NIMH Treatment of Depression Collaboratic Research Program: Where we began and where we are now. In: A. E. Bergin und S. L. Garfield (Hrsg.), Handbook of psychotherapy and behavior change (4. Ausgabe). New York: Wiley, 114-135.

Ferenczi, S., Rank, O. (1984 [1924]): Entwicklungsziele der Psychoanalyse. Zur Wechselbeziehung von Theorie und Praxis. In. S. Ferenczi (Hrsg.), Bausteine zur Psychoanalyse, Bd. III: Arbeiten aus den Jahren 1908-1933. Bern, Stuttgart, Wien: Huber, 220-244.

Ferenczi, S. (1984 [1926]): Aktuelle Probleme der Psychoanalyse. Bausteine zur Psychoanalyse, Bd. III: Arbeiten aus den Jahren 1908-1933: Bern, Stuttgart, Wien: Huber, 332-346.

Ferenczi, S. (1984 [1931]): Kinderanalysen mit Erwachsenen. Bausteine zur Psychoanalyse, Bd. III: Arbeiten aus den Jahren 1908-1933. Bern, Stuttgart, Wien: Huber 490-510.

Freud, S. (1952a [1895]): Studien über Hysterie. GW I. Frankfurt a. M.: Fischer Verlag.

Freud, S. (1952b [1919]): Wege der psychoanalytischen Therapie. GW XII. Frankfurt a. M.: Fischer Verlag.

Grawe, K., Donati, R. und Bernauer, F. (1994): Psychotherapie im Wandel-Von der Konfession zur Profession. Göttingen: Hogrefe.

Interpharma (2007): Gesundheitswesen Schweiz. Ausgabe 2007. Basel: Interpharma.

Psychotherapie 
Leichsenring F. u. Leibing, E. (2003): The effectiveness of psychodynamic psychotherapy and cognitiv-behavioral therapy in personality disorders. A metaanalysis. American Journal of Psychiatry 20, 34-38.

Malan, D. H. (1965): Psychoanalytische Kurztherapie. Stuttgart: Klett.

Margraf, J. (Hrsg.) (2000a): Lehrbuch der Verhaltenstherapie. Band 1: Grundlagen, Diagnostik, Verfahren, Rahmenbedingungen. 2., vollständig überarbeitete und erweiterte Auflage. Berlin: Springer.

Margraf, J. (Hrsg.) (2000b): Lehrbuch der Verhaltenstherapie. Band 2: Störungen, Glossar. 2., vollständig überarbeitete und erweiterte Auflage. Berlin: Springer.

May, U. (2007): Neunzehn Patienten in Analyse bei Freud (1910-1920): Teil I: Zur Dauer von Freuds Analysen. Psyche-Zeitschrift für Psychoanalyse und ihre Anwendungen, 61(6), 590-625.

Richter, H.E. (2005): Psychotherapie Gestern, Heute und Morgen. Festvortrag anlässlich des 25-jährigen Jubiläums des Schweizer Psychotherapeutinnen und Psychotherapeuten Verbandes SPV am 24. September 2004 in Zürich. À Jour - Aktuelles für SPV-Mitglieder, 11-16.

Smith, M.L., Glass, G.V. and Miller, T.I. (1980): The benefits of psychotherapy. Baltimore: Johns Hopkins University Press.

Stekel, W. (1938): Technik der psychoanalytischen Psychotherapie. Bern: Huber.

Sturny, I. \& Hell, D. (2007): Psychiatrie, Psychotherapie, Psychologie. In: G. Kocher, W. Oggier (Hrsg.), Gesundheitswesen Schweiz 2007-2009. Eine aktuelle Übersicht. Bern: Verlag Hans Huber, 291-304).

Thomä, H. \& Kächele, H. (1996): Lehrbuch der psychoanalytischen Therapie. Band 1: Grundlagen. 2. überarb. Auflage. Berlin: Springer.

Winnicott, D.W. (1965/1993): Reifungsprozesse und fördernde Umwelt. Frankfurt/M.: Fischer. 